\title{
RISK EXCHANGE WITH DISTORTED PROBABILITIES
}

\author{
BY
}

Andreas Tsanakas And Nicos Christofides ${ }^{1}$

\begin{abstract}
An exchange economy is considered, where agents (insurers/banks) trade risks. Decision making takes place under distorted probabilities, which are used to represent either rank-dependence of preferences or ambiguity with respect to real-world probabilities. Pricing formulas and risk allocations, generalising the results of Bühlmann $(1980,1984)$ are obtained via the construction of aggregate preferences from heterogeneous agents' utility and distortion functions. This involves the introduction of a novel 'collective ambiguity aversion' coefficient. It is shown that probability distortion changes insurers' behaviour, who trade not only to share the aggregate market risk, but are also found to bet against each other. Moreover, probability distortion tends to increase the price of insurance (increase asset returns). While the cases of rank-dependence and ambiguity are formally similar, an important distinction emerges as for rank-dependent preferences equilibria are determinate, while for ambiguity they are generally indeterminate.
\end{abstract}

\section{INTRODUCTION}

Equilibrium asset pricing models of financial and insurance markets have been extensively studied in the economics, financial and actuarial literature. A pioneering paper in the subject area is by Borch (1962), whose approach was continued by Bühlmann's $(1980,1984)$ celebrated pricing models. Useful overviews are provided by Duffie (2001) and Aase (1993, 2002). The purpose of this paper is to provide asset pricing and risk sharing models, which address two distinct issues that are not present in the classic insurance/asset pricing literature. The first concerns the violations of utility theory frequently observed in practice, which can be explained by a distorted perception of probability by economic agents (Quiggin, 1993). The second issue is the presence of Knightian uncertainty or ambiguity in financial markets. It was argued by Knight (1921) that there persists significant uncertainty in markets, not only with respect to the future

1 The authors are grateful to an anonymous referee for insightful suggestions that significantly improved the paper. 
states of the world, but also with respect to the probabilities of those states. Besides the main goal of determining asset prices in the above situations, we are interested in asking questions such as: What are the characteristics of equilibrium allocations when agents operate under a distorted probability and do not conform to the expected utility paradigm? How are market prices affected by ambiguity? Do diverging beliefs create additional incentives for trading? How can one express ambiguity on a market-aggregate, rather than agent-specific level?

The above issues are studied within the analytical framework provided by distorted probabilities. Distorted probabilities arise from the application of non-linear functions on probability measures and allow three distinct interpretations. The first is in the context of preferences modelled by Rank-Dependent Expected Utility (RDEU) theory (Quiggin, 1982; 1993), where the probability distortion is seen as reflecting the effect of distorted perception of probability by economic agents. Experimental evidence suggests that such a distortion is a common feature of decision making under risk and leads to violations of the expected utility paradigm, e.g. to phenomena such as the Allais paradox, the common ratio effect and preference reversal (Quiggin, 1993). Distorted probabilities can also be shown to give rise to a set of probability measures or 'priors', whose presence can be seen as a representation of Knightian uncertainty. This second interpretation of probability distortion gives rise to an economic decision model under ambiguity known as Choquet Expected Utility (CEU) (Schmeidler, 1989). A third interpretation, not discussed in detail in this paper, relates to the use of distorted probabilities in constructing functionals (Denneberg (1990), Wang (1996)) that are consistent with the coherence axioms of Artzner et al. (1999) for risk measures used to set regulatory capital requirements.

Apart from reflecting individual preferences that are inconsistent with expected utility theory, distorted probabilities can be employed to explain particular economic phenomena that the classical model fails to address. Classic utility theory implies that agents' risk allocations are shares of the market risk portfolio. However this is not the case in practice for a variety of reasons, including market incompleteness and asymmetric information. The framework of distorted probability shows that a 'betting' behaviour produced by rank-dependent preferences and ambiguity could be an additional reason behind this. Another important phenomenon for which our framework can provide explanation is the equity premium puzzle (Mehra and Prescott, 1985), that is, the observation that asset returns are higher than what a utility-based model would predict. Moreover, it is noted that the emergence of risk sensitive regulation in recent years (e.g. Basle, 2003) produces a new set of imperatives for financial institutions. As distorted probabilities are an effective tool for producing well behaved risk measures for setting capital requirements, it has been argued that a risk takers decision problem should include both utility and probability distortion components (Tsanakas and Desli, 2003). It is noted that in the latter interpretation, probability distortion is introduced in a normative rather than descriptive way, as it reflects regulatory imperatives rather than individual preferences. 
Equilibrium models where distorted probabilities are used for representing agents' preferences have been studied in the literature, usually with the interpretation of CEU as a model for ambiguity. Dow and Werlang (1992) studied the problem of portfolio choice under ambiguity and showed that when an agent can invest into one risky and one risk-free asset, there is an interval of (exogenously given) prices, under which no trade takes place in the risky asset. Epstein and Wang (1994) and Chen and Epstein (2002) develop dynamic representative agent asset pricing models, using generalisations of Gilboa and Schmeidler's (1989) maxmin expected utility model. No-trade intervals and indeterminacy of the resulting equilibria, are features of these models too. Characterisations of Pareto optimal allocations are given in Chateauneuf et al. (2000) and Dana (2002).

In this paper we study an risk exchange economy, similar to the one considered by Buhlmann $(1980,1984)$, with the difference that economic agents' decision-making takes place under a distorted probability. Each agent is thus characterised by a utility and a distortion function. We determine equilibrium prices and risk allocations, thus providing the basis for an insurance/asset pricing model. In the context of the risk exchange the different interpretations of distorted probability are treated to some extent concurrently, due to the formal similarity between the models. Thus we discuss equilibria and determine price functionals, when agents' preferences are characterised by RDEU and when agents are ambiguous about the probabilities of future events (CEU). It is noted that as the paper is primarily about Pareto optima, conditions for existence of equilibrium are not discussed. The particular analytical tool that enables us to solve the posed preference maximisation problems is the concept of 'quantile derivatives' (Tasche, 2000), which proves useful for the differentiation of functionals involving distorted probabilities.

In equilibrium models with heterogeneous agents, a standard technique for calculating prices is to construct the preferences of a fictional 'representative agent' (e.g. Duffie, 2001) and then determine prices as marginal costs to this agent. We carry out a construction of aggregate preferences under distorted probabilities. Such preferences are expressed in terms of two quantities which we call 'collective risk and ambiguity aversions'. While the collective risk aversion is the usual (e.g. Wilson, 1968) inverse of the sum of agents' Arrow-Pratt risk tolerances, the notion of collective ambiguity aversion is introduced in this paper. If we interpret collective ambiguity aversion and the associated distortion function as a quantifier of ambiguity at market level, it is shown that ambiguity, as opposed to ordinary risk, cannot by 'diversified away' by trading in a market.

As one of our aims is to present a pricing model, we obtain explicit and transparent pricing formulas for traded risks, as well as for the equilibrium risk allocations. The price density depends on two factors, one relating to collective risk and one to collective ambiguity aversion, and can be seen to be a generalisation of the pricing formulas obtained by Bühlmann (1980, 1984). It is shown that the effect of Knightian uncertainty is to inflate asset returns (equivalently 
to increase the price of insurance). As already observed by Epstein and Wang (1994), this implies that ambiguity is one of the candidate explanations for the equity premium puzzle (Mehra and Prescott, 1985). It is a contribution of this study that such reduction in asset may also be due to rank dependence of preferences.

Agents' equilibrium risk allocations also consist of two parts, relating to risk and ambiguity aversion respectively. If we interpret distortion functions as generators of ambiguity, the latter part of the allocation only occurs due to agents' diverging beliefs. It is shown that, given the presence of aggregate risk in the market, risk aversion causes the agents to share that risk, while ambiguity provides an incentive to bet against each other. If all agents are characterised by the same distortion function, such betting behaviour vanishes and trading takes place only in relation with sharing the aggregate market risk. It is noted that betting behaviour has been produced by previous models, such as Wilson's (1968), which considers diverging beliefs in the context of Savage expected utility. The present paper shows that rank dependence of preferences as well as diverging beliefs cause incentives for betting.

Equilibrium models where agents are characterised either by a distorted perception of probability or by ambiguity are solved concurrently. However, an important difference arises relating to the determinacy of the equilibrium. In the case of RDEU the equilibrium calculated in the paper is completely determined, while in the case of CEU it is not. Mathematically, this follows from the fact that ambiguity is not represented uniquely by a distortion function; different representations give rise to different equilibria. While in the equilibrium with RDEU it is implicitly assumed that all agents agree on a probability, in the case of ambiguity agreement on a (reference) probability measure would be meaningless. On the other hand, if the agents' ambiguous beliefs are characterised by the same sets of probabilities, in effect an agreement becomes possible and the indeterminacy vanishes.

The structure of the paper is as follows. In Section 2 we introduce the preference functionals used in the paper, along with a discussion of risk and ambiguity aversion, as defined for the purposes of the present investigation. In Section 3 the equilibrium models for Rank Dependent and Choquet Expected Utilities are presented. Pricing and risk allocation formulas are given, and the effect of ambiguity, the (in)determinacy of equilibrium, and the concept of collective preferences, are discussed. Conclusions from the paper are summarised in Section 4.

\section{Preferences}

\subsection{Preference functionals}

A one-period economy is considered. At time 0 economic agents (e.g. financial institutions, insurance companies) make decisions concerning their consumption of assets and liabilities with random payoffs. At some fixed future time $t$ 
the state of the world is revealed and gains and losses are realised. A probability space $\left(\Omega, \mathbb{P}_{0}, \mathcal{F}\right)$ is defined, where $\Omega$ is the set of all possible states of the world at time $t, \mathbb{P}_{0}$ is a probability measure (which will be interpreted according to the context as either the actuarial 'real-world' probability or just a reference measure), and $\mathcal{F} \subset 2^{\Omega}$ is a $\sigma$-algebra with respect to which random variables are measurable, representing the amount of information available to agents at time $t$. We consider a set, $X$, of square-integrable random variables on this probability space, which represent investment opportunities available to the market agents. For technical reasons we assume that elements of $X$ have continuous conditional densities in the sense of Tasche (2000). ${ }^{2}$ Elements of $X$ are henceforth called positions. We denote by $E[\cdot]$ the expectation operator under $\mathbb{P}_{0}$ and use the notation $S_{X}(x)=\mathbb{P}_{0}(X>x)$ for the decumulative (survival) distribution function of $X \in X$.

For each market agent, a preference relation ' $\succeq$ ' is defined on $X$, associated with a preference functional $V: X \mapsto \mathbb{R}$, i.e. $V(X) \geq V(Y) \Leftrightarrow X \succeq Y$. (It is in fact the owners of the financial institutions / insurance companies that are endowed with preferences rather than the companies themselves. Individuals owning the companies' random portfolios have preferences over end-of-period consumption, which in the present one-period setting corresponds to random wealth). The preference functional is given by:

$$
V_{u, h}(X)=\int_{-\infty}^{0}\left(h\left(S_{u(X)}\right)-1\right) d x+\int_{0}^{\infty} h\left(S_{u(X)}\right) d x,
$$

where $u$ is an increasing and concave utility function and $h$ is an increasing and convex probability distortion function with $h(0)=0$ and $h(1)=1$. The set function $h\left(\mathbb{P}_{0}\right)$ is called a distorted probability. The above preference functional emerges as a generalisation of the von Neumann-Morgenstern (1947) expected utility operator; in fact, when $h$ is linear, equation (1) reduces to an expected utility. There are two possible interpretations of the effect of the probability distortion $h$.

The first interpretation, in the context of Rank-Dependent Expected Utility (Quiggin, 1982), is a behavioral one. Under such a light, the probability distortion $h$ is related to the perception of probability by an economic agent. It has been observed (e.g. Quiggin, 1993) that agents often tend to overstate the probability of adverse events. Note that an adverse event is not understood here by the value itself of the random variable $X$, but by its rank among all possible outcomes. It is noted Quiggin's (1993) development considers a different formulation of the preference functional: $V_{u, h}(X)=\int u(x) d\left(g \circ F_{X}(x)\right)$. This can be derived from (1) by integration by parts and setting $g(s)=1-h(1-s)$. The reason for using the slightly more complicated expression (1) is to achieve consistency with Choquet Expected Utility discussed below.

2 The assumption of continuity is a sufficient condition for the differentiability of preference functionals, see Lemma 6. For an alternative approach see Carlier and Dana (2002). 
An alternative interpretation relates to the study of preferences under Knightian uncertainty, in the sense of ambiguity with respect to the probability distribution of the underlying risks. The distorted probability can be viewed as a set function $\gamma=h\left(\mathbb{P}_{0}\right)$. Moreover, when $h$ is increasing and convex, the set function $\gamma$ is supermodular, i.e. $\gamma(A \cup B)+\gamma(A \cap B) \geq \gamma(A)+\gamma(B), A, B \in \mathcal{F}$ (Denneberg, 1994). Then, the preference functional (1) is re-expressed as the Choquet integral:

$$
V_{u, \gamma}(X)=\int u(X) d \gamma=\int_{-\infty}^{0}(\gamma(u(X)>x)-1) d x+\int_{0}^{\infty} \gamma(u(X)>x) d x .
$$

Choquet integrals are defined with respect to monotone set functions (or 'capacities') instead of additive measures (Choquet (1954), Denneberg (1994)). The preference functional (2) can be derived from a set of axioms (Schmeidler, 1989), and is called a Choquet Expected Utility.

The relationship of Choquet expected utility to Knightian uncertainty can be seen via the representation of the supermodular set function $\gamma$ and the respective preference functional (2) through sets of probability measures (Denneberg, 1994):

$$
\gamma(A)=\inf _{\mathbb{P} \geq \gamma} \mathbb{P}(A), A \in \mathcal{F}, \int u(X) d \gamma=\inf _{\mathbb{P} \geq \gamma} E_{\mathbb{P}}[X], X \in X .
$$

Thus, the preference functional can be understood as the minimal expected utility with respect to a set of probability measures induced by the set function $\gamma$. The fact that a set of probability measures is used instead of only one reflects ambiguity with respect to the actual probability distribution of the risk $X \in X$. That expected utility is evaluated at the infimum with respect to that set of measures reflects the aversion of economic agents to such ambiguity. We note that this interpretation of the distortion function, the probability measure $\mathbb{P}_{0}$ is no more a 'real-world' probability but just a reference measure used in representing the capacity, $\gamma$, via a distorted probability, $h\left(\mathbb{P}_{0}\right)$. Technical conditions under which such a representation is possible are studied by Gilboa (1985) and Wanget al. (1997).

We conclude this section by stating the set of assumptions on utility and distortion functions that are used throughout the paper. Utility functions are strictly increasing, strictly concave, continuous and twice differentiable. Distortion functions are strictly increasing, strictly convex, continuous and twice differentiable. Furthermore, we assume throughout that the economic agents characterised by CEU have capacities which can be expressed by distorted probabilities.

\subsection{Risk aversion}

In the context of RDEU, the effect of the utility and distortion functions is quite different, though difficult to disentangle. Considering the relationship between RDEU and risk aversion as expressed through the concept of mean 
preserving increase in risk (MPIR) (Rothschild and Stiglitz, 1970), Chew et al. (1987), have shown that: (i) a preference relation displays aversion to MPIR, in the sense that every mean preserving increase in risk reduces the value of the preference functional, if and only if the utility function is concave and the distortion function is convex; and (ii) one preference relation is more averse to MPIR than another if and only if the utility and distortion functions of the former are respectively concave and convex transformations of those of the latter.

On the other hand, in the context of Schmeidler's (1989) model of preferences under ambiguity, the convexity of the capacity $\gamma$ (and therefore of the distortion function) is a mathematical precondition for representing the preference functional as a minimal expected utility with respect to a set of probability measures. In that sense convexity of the distortion function reflects 'ambiguity aversion'.

The concavity of the utility function is usually characterised through the Arrow-Pratt coefficient of risk aversion:

Definition 1. The coefficient of risk aversion associated with a (twice differentiable) utility function $u$ is defined as

$$
\rho(x)=-\frac{u^{\prime \prime}(x)}{u^{\prime}(x)} .
$$

For the purposes of this investigation, we proceed to characterizing the convexity of a distortion function in a very similar way:

Definition 2. The coefficient of ambiguity aversion associated with a (twice differentiable) distortion function $h$ is defined as

$$
\tau(s)=\frac{h^{\prime \prime}(s)}{h^{\prime}(s)} .
$$

The term 'ambiguity aversion' relates to the interpretation of the distortion function as a way of generating a set of probability measures; as will be shown below, the more convex $h$ is, the larger is the set of measures $\{\mathbb{P}: \mathbb{P}(A) \geq$ $h\left(\mathbb{P}_{0}(A)\right) \forall A \in \mathcal{F}$ \} induced by it and thus the higher the ambiguity surrounding the probability distribution. Even though in this paper the distortion function is not used exclusively in the CEU context, we will use for simplicity the term 'ambiguity aversion' throughout. It is noted that the differential equations (4) and (5) can be solved to determine uniquely a utility (up to an affine transformation) and a distortion function.

The above definitions of risk and ambiguity aversion coefficients relate to the comparison by Chew et al. (1987) of preference functional in terms of their aversion to MPIR, as shown below.

Lemma 1. (i) An agent characterised by a utility function, $u$, and a distortion function, $h$, is averse to MPIR if and only if the associated risk and ambiguity aversion coefficients are non-negative, i.e. $\rho(x) \geq 0 \forall x \in \mathbb{R}$ and $\tau(s) \geq 0, \forall s \in[0,1]$. 
(ii) An agent characterised by utility and distortion functions, $u_{1}, h_{1}$, respectively is more averse to MPIR than another with utility and distortion functions, $u_{2}, h_{2}$, if and only it holds for the associated risk and ambiguity aversion coefficients that $\rho_{1}(x) \geq \rho_{2}(x) \forall x \in \mathbb{R}$ and $\tau_{1}(s) \geq \tau_{2}, \forall s \in[0,1]$.

Proof:

(i) Follows trivially from Chew et al. (1987), as positivity of the risk and ambiguity aversion coefficients guarantee the concavity and convexity of the utility and distortion functions respectively.

(ii) We must show that the condition $\rho_{1}(x) \geq \rho_{2}(x) \forall x \in \mathbb{R}$ (resp. $\tau_{1}(s) \geq \tau_{2}, \forall s \in$ $[0,1])$ is equivalent to $u_{1}$ (resp. $h_{1}$ ) being a concave (resp. convex) transformation of $u_{2}$ (resp. $h_{2}$ ).

If $u_{1}(x)=c\left(u_{2}(x)\right)$, where $c$ is an increasing and concave function,

$$
u_{1}^{\prime}(x)=c^{\prime}\left(u_{2}(x)\right) u_{2}^{\prime}(x), u_{1}^{\prime \prime}(x)=c^{\prime \prime}\left(u_{2}(x)\right)\left(u_{2}^{\prime}(x)\right)^{2}+c^{\prime}\left(u_{2}(x)\right) u_{2}^{\prime \prime}(x)
$$

Thus

$$
-\frac{u_{1}^{\prime \prime}(x)}{u_{1}^{\prime}(x)}=-\frac{c^{\prime \prime}\left(u_{2}(x)\right) u_{2}^{\prime}(x)}{c^{\prime}\left(u_{2}(x)\right)}-\frac{u_{2}^{\prime \prime}(x)}{u_{2}^{\prime}(x)} \Rightarrow \rho_{1}(x) \geq \rho_{2}(x)
$$

Conversely, if $\rho_{1}(x) \geq \rho_{2}(x) \Leftrightarrow \rho_{1}(x)=\rho_{2}(x)+f(x), f(x) \geq 0$, then we can define the increasing and concave function:

$$
c(x)=\int_{-\infty}^{x} \exp \left(-\int_{-\infty}^{u_{2}^{-1}(y)} f(t) d t\right) d y,
$$

It is then easy to show that

$$
f(x)=-\frac{c^{\prime \prime}\left(u_{2}(x)\right) u_{2}^{\prime}(x)}{c^{\prime}\left(u_{2}(x)\right)},
$$

which, given $\rho_{1}(x)=\rho_{2}(x)+f(x)$, yields $u_{1}(x)=c\left(u_{2}(x)\right)$.

The proof for the distortion functions is the same.

Finally, in the context of ambiguity represented by distorted probabilities, it can be shown that comparing the ambiguity aversion coefficient associated with two agents provides a comparison of the ambiguity characterizing each.

Lemma 2. Let two agents' preferences be characterised by Choquet Expected Utility and their respective supermodular capacities can be represented as $h_{1}\left(\mathbb{P}_{0}\right)$ 
and $h_{2}\left(\mathbb{P}_{0}\right)$. Define the ambiguity aversions $\tau_{1}, \tau_{2}$ by (5) and the sets $\mathcal{P}_{1}=\{\mathbb{P}$ : $\left.\mathbb{P}(A) \geq h_{1}\left(\mathbb{P}_{0}(A)\right) \forall A \in \mathcal{F}\right\}$ and $\mathcal{P}_{2}=\left\{\mathbb{P}: \mathbb{P}(A) \geq h_{2}\left(\mathbb{P}_{0}(A)\right) \forall A \in \mathcal{F}\right\}$ representing the agents' ambiguity. Then, $\tau_{2}(s) \leq \tau_{1}(s) \forall s \in[0,1]$ implies that $\mathcal{P}_{2} \subseteq \mathcal{P}_{1}$.

\section{Proof:}

From Lemma 1ii) it can be seen that $\tau_{1}(s) \geq \tau_{2}(s)$ implies that $h_{1}(s)=c\left(h_{2}(s)\right)$, where $c$ is an increasing convex function with $c(0)=0, c(1)=1$. This in turn implies that $h_{1}(s) \leq h_{2}(s) \forall s \in[0,1]$ which yields $\mathcal{P}_{2} \subseteq \mathcal{P}_{1}$.

\section{RISK EXCHANGE}

\subsection{General setup}

Let $n$ agents, standing for financial institutions ((re)insurance companies, banks etc), be participating in an exchange economy, similar to the one defined by Borch (1962) and Bühlmann (1980,1984). Each holds an initial endowment $X_{i} \in X, i=1, \ldots, n$ (random assets and liabilities including cash), which can be traded in the exchange. Let $\mathcal{F}$ be the $\sigma$-algebra generated by the initial endowments $X_{i}, i=1, \ldots, n$. Agents can acquire through trading any position $Y \in X$ that is measurable with respect to $\mathcal{F}$, that is, the positions available to traders are restricted to functions of the $X_{i}$ 's. Additionally we assume that a safe asset $\mathbf{1}_{\Omega}$ with unit price and unit payoff is traded in the market; this implies zero interest rates. ${ }^{3}$ We assume that market prices are given by a linear functional $\pi(X)=E[\zeta X]$, where $\zeta \in L^{2}\left(\Omega, \mathbb{P}_{0}, \mathcal{F}\right)$. The price of the safe asset is 1 , hence:

$$
\pi\left[\mathbf{1}_{\Omega}\right]=1 \Rightarrow E[\zeta]=1 .
$$

Agents are characterised by preference functionals of the form (1). Each agent is equipped with a strictly increasing and concave utility function $u_{i}$ and a strictly increasing and convex probability distortion $h_{i}, i=1, \ldots, n$, both $u_{i}$ and $h_{i}$ being continuous and twice differentiable. We denote the $i$ th agent's preference functional as $V_{i}$. The $i$ th agent decides on his optimal investment by maximizing his preference functional, subject to a budget condition:

$$
\max _{Y_{i}} V_{i}\left(Y_{i}\right), \text { such that } \pi\left(Y_{i}\right) \leq \pi\left(X_{i}\right) .
$$

As discussed in Section 2.1 these preference functionals can be associated either with RDEU or with CEU. Due to the formal similarity of these two models, they

3 We note that this collection of available positions includes nonlinear functions of the initial endowments and thus refers to an infinite-dimensional commodity space. The richness of this class of traded assets is necessary for the complete market setting implicitly assumed here, due to the fact that the probability space is also infinite dimensional. 
are treated concurrently in the sequel. However, an important difference arises relating to the determinacy of equilibrium, which is discussed in Section 3.5. Furthermore, an implicit assumption used in the sequel is that, if the preference functional is associated with Choquet expected utility, all agents' capacities can be expressed as distortions of the same probability measure. This has two consequences. One is that all capacities have the same null-sets. The other is that the sets of probability measures associated with each agent have a nonempty intersection (it is easy to see that the reference measure will belong to all those sets, e.g. $\mathbb{P}_{0}(A) \geq h\left(\mathbb{P}_{0}(A)\right), A \in \mathcal{F}$.

\subsection{Necessary conditions for equilibrium}

We define the aggregate risk in the market as $Z=\sum_{j=1}^{n} X_{j}$. The economy will be at equilibrium if and when all agents have solved their preference maximisation problem (11) and the market has cleared:

$$
\sum_{j=1}^{n} Y_{j}=Z
$$

The optimisation problem (11) has Lagrangian:

$$
V_{i}\left(Y_{i}\right)-\lambda_{i}\left(\pi\left(Y_{i}\right)-\pi\left(X_{i}\right)\right) .
$$

The following lemma yields a necessary condition for equilibrium, which can be viewed as a generalised version of Borch's (1962) characterisation of Pareto optima.

Lemma 3. (i) At equilibrium each agent's risk allocation, $Y_{i}$, is related to the price density, $\zeta$, via the relationship

$$
u_{i}^{\prime}\left(Y_{i}\right) h_{i}^{\prime}\left(S_{Y_{i}}\left(Y_{i}\right)\right)=\lambda_{i} \zeta, i=1, \ldots, n .
$$

(ii) The risk allocations to the agents, $Y_{1}, Y_{2}, \ldots, Y_{n}$ are comonotonic random variables.

Proof:

(i) To solve the maximisation problem (11) we proceed using some standard methodology from variational calculus. For $N \in \mathcal{X}$ we define $f(\beta)=V_{i}\left(Y_{i}+\right.$ $\beta N)-\lambda_{i}\left(\pi\left(Y_{i}+\beta N\right)-\pi\left(X_{i}\right)\right)$. In order that the objective function of (11) achieves an optimum at $Y_{i}$ it must be, $f^{\prime}(0)=0, \forall N \in X$. From Lemma 6 we obtain:

$$
f^{\prime}(\beta)=E\left[N u_{i}^{\prime}\left(Y_{i}+\beta N\right) h_{i}^{\prime}\left(S_{Y_{i}+\beta N}\left(Y_{i}+\beta N\right)\right)\right]-\lambda_{i} \pi(N) .
$$


Thus:

$$
f^{\prime}(0)=E\left[N u_{i}^{\prime}\left(Y_{i}\right) h_{i}^{\prime}\left(S_{Y_{i}}\left(Y_{i}\right)\right)\right]-\lambda_{i} \pi(N)=0 \forall N \in X,
$$

which yields condition (14).

(ii) Consider equation (14). Since both $u_{i}$ and $h_{i}$ are strictly increasing, $\lambda_{i}>0$. Consider now the function $\eta_{i}(x)=\left(1 / \lambda_{i}\right) u_{i}^{\prime}(x) h_{i}^{\prime} \circ S_{Y_{i}}(x)$. The first derivative of $\eta_{i}$ is strictly negative

$$
\eta_{i}^{\prime}(x)=\frac{1}{\lambda_{i}}\left(u_{i}^{\prime \prime}(x) h_{i}^{\prime} \circ S_{Y_{i}}(x)-u_{i}^{\prime}(x) h_{i}^{\prime \prime} \circ S_{Y_{i}}(x) f_{Y_{i}}(x)\right)<0,
$$

since the functions $u_{i}^{\prime} h_{i}^{\prime}$ are strictly decreasing and increasing respectively. Thus $\eta_{i}$ is strictly decreasing. Therefore its inverse $\eta_{i}^{-1}$ exists and is also strictly decreasing. We observe that all random variables $Y_{i}=\eta_{i}^{-1}(\zeta)$ are strictly decreasing functions of the random variable $\zeta$. Hence $Y_{1}, Y_{2}, \ldots, Y_{n}$ are comonotonic.

Comonotonic risks are characterised by the strongest form of positive statistical dependence. An economic interpretation of comonotonic risks is that they cannot be used as hedges for each other (Yaari, 1987). The fact that the final positions $Y_{i}$ are comonotonic has the interpretation that agents have ridded themselves of the individual risk embedded in their initial endowments $X_{i}$ and are left only with the market's systemic risk. Thus, our model is consistent with a well known tenet of capital asset pricing. Moreover, it has been shown that comonotonicity of the risk allocations $Y_{i}$ is a precondition for efficient spreading of the market risk to the agents (Landsberger and Meilijson, 1994).

Comonotonicity of the random variables $Y_{1}, Y_{2}, \ldots, Y_{n}$ has two important consequences which will prove useful in the sequel. These are summarised below.

Lemma 4. The following properties of comonotonic random variables hold:

a) The comonotonic random variables $Y_{i}, i=1, \ldots, n$ are also comonotonic to (increasing functions of) their sum $Z$.

b)

$$
F_{Y_{i}}\left(Y_{i}\right)=F_{Z}(Z)=U \text { a.s., } \forall i=1, \ldots, n,
$$

where $U$ is uniformly distributed on the unit interval.

Proof: E.g. Dhaene et al. (2002).

\subsection{Solution for exponential utility and distortion functions}

Before proceeding with the calculation of equilibrium prices for more general utility and distortion functions, we study the case of exponential utility and 
distortion. This situation gives rise to simple and transparent solutions, as well as providing a generalisation of Bühlmann's (1980) pricing formula and a distorted probability version of the well-known Esscher transform.

Let each agent have an exponential utility function with risk aversion $\rho_{i}>0$ and an exponential distortion function $h_{i}$ with ambiguity aversion $\tau_{i}>0$ :

$$
u_{i}(x)=\frac{1}{\rho_{i}}\left(1-e^{-\rho_{i} x}\right), \quad h_{i}(s)=\frac{e^{\tau_{i} s}-1}{e^{\tau_{i}}-1} .
$$

The first and second derivatives of these functions are:

$$
\begin{aligned}
& u_{i}^{\prime}(x)=e^{-\rho_{i} x}>0, u_{i}^{\prime \prime}(x)=-\rho_{i} e^{-\rho_{i} x}<0 . \\
& h_{i}^{\prime}(s)=\frac{\tau_{i} e^{\tau_{i} s}}{e^{\tau_{i}}-1}>0, h_{i}^{\prime \prime}(s)=\frac{\tau_{i}^{2} e^{\tau_{i} s}}{e^{\tau_{i}}-1}>0 .
\end{aligned}
$$

In the sequel, the following rewriting of $h_{i}^{\prime}\left(S_{Y_{i}}\left(Y_{i}\right)\right)$ will also be used:

$$
h_{i}^{\prime}\left(S_{Y_{i}}\left(Y_{i}\right)\right)=\frac{e^{-\tau_{i} F_{Y_{i}}\left(Y_{i}\right)}}{E\left[e^{-\tau_{i} F_{Y_{i}}\left(Y_{i}\right)}\right]} .
$$

We now define two quantitities which prove useful in the sequel.

Definition 3. In the risk exchange with exponential utility and distortion functions, the collective risk aversion, $\rho$, and the collective ambiguity aversion, $\tau$, are defined by the equations:

$$
\frac{1}{\rho}=\sum_{j=1}^{n} \frac{1}{\rho_{j}}, \quad \tau=\rho \sum_{j=1}^{n} \frac{\tau_{j}}{\rho_{j}} .
$$

The collective risk and ambiguity aversions are treated for the time being only as notational simplifications. Their meaning will be discussed in Section 3.6. We note here that the formula for collective risk aversion is well known (e.g. Bühlmann, 1980), while the formula for collective ambiguity aversion is being introduced in this paper.

The following result provides a simple formula for the equilibrium price density, $\zeta$.

Proposition 1. In the risk exchange with exponential utilities and distortions the equilibrium price density, $\zeta$, has the form:

$$
\zeta=\frac{e^{-\rho Z-\tau F_{Z}(Z)}}{E\left[e^{-\rho Z-\tau F_{Z}(Z)}\right]} .
$$


Proof: Condition (14) for equilibrium that was derived earlier yields:

$$
e^{-\rho_{i} Y_{i}} h_{i}^{\prime}\left(S_{Y_{i}}\left(Y_{i}\right)\right)=\lambda_{i} \zeta \Rightarrow Y_{i}=\frac{1}{\rho_{i}} \ln \left(h_{i}^{\prime}\left(S_{Y_{i}}\left(Y_{i}\right)\right)\right)-\frac{1}{\rho_{i}} \ln (\zeta)-\frac{1}{\rho_{i}} \ln \left(\lambda_{i}\right) .
$$

By summing both sides of the above equation over $i$ and taking into account the clearing condition (12) we obtain:

$$
Z=\sum_{j=1}^{n} \frac{1}{\rho_{j}} \ln \left(h_{j}^{\prime}\left(S_{Y_{j}}\left(Y_{j}\right)\right)\right)-\sum_{j=1}^{n} \frac{1}{\rho_{j}} \ln (\zeta)-\sum_{j=1}^{n} \frac{1}{\rho_{j}} \ln \left(\lambda_{j}\right) .
$$

The first term of the right-hand side becomes:

$$
\begin{aligned}
& \ln \prod_{j=1}^{n} h_{j}^{\prime}\left(S_{Y_{j}}\left(Y_{j}\right)\right)^{\frac{1}{\rho_{j}}} \stackrel{(22)}{=} \ln \frac{\prod_{j=1}^{n} e^{-\frac{\tau_{j}}{\rho_{j}} F_{Y_{j}}\left(Y_{j}\right)}}{\prod_{j=1}^{n} E\left[e^{-\tau_{j} F_{Y_{j}}\left(Y_{j}\right)}\right]^{\frac{1}{\rho_{j}}}}= \\
& \stackrel{(18)}{=} \ln e^{-\sum_{j=1}^{n} \frac{\tau_{j}}{\rho_{j}} U}-\ln \prod_{j=1}^{n} E\left[e^{-\tau_{j} U}\right]^{\frac{1}{\rho_{j}}} .
\end{aligned}
$$

Now, by putting together equations (26), (27) and (23) we obtain:

$$
Z=-\frac{\tau}{\rho} U-\ln \prod_{j=1}^{n} E\left[e^{-\tau_{j} U}\right]^{\frac{1}{\rho_{j}}}-\frac{1}{\rho} \ln (\zeta)-\sum_{j=1}^{n} \frac{1}{\rho_{j}} \ln \left(\lambda_{j}\right) .
$$

We set the constant, $K=-\rho\left(\ln \prod_{j=1}^{n} E\left[e^{-\tau_{j} U}\right]^{\frac{1}{\rho_{j}}}+\sum_{j=1}^{n} \frac{1}{\rho_{j}} \ln \left(\lambda_{j}\right)\right)$. Then equation (28) becomes:

$$
\rho Z+\tau U=K-\ln \zeta \Rightarrow \zeta=e^{-\rho Z-\tau U} e^{K} .
$$

Since we have assumed that there exists in the market a risk-free asset $\mathbf{1}_{\Omega}$ with unit price, from (10) we obtain:

$$
E[\zeta]=1 \stackrel{(29)}{\Rightarrow} E\left[e^{-\rho Z-\tau U} e^{K}\right]=1 \Rightarrow e^{K}=E\left[e^{-\rho Z-\tau U}\right]^{-1} .
$$

Substituting $\exp (K)$ in (29), we obtain formula (24) for the price density.

Note that formula (24) is a generalisation of the Esscher transform, which was obtained by Bühlmann (1980), who studied a market model where agents' preferences are characterised by exponential utility functions. The probability weighting factor $\exp (-\rho Z)$ in the price density associates the price of a traded position with the random value of the market portfolio $Z$. The fact that it is a decreasing function of $Z$ has the interpretation that a position, which is likely 
to assume a high value when $Z$ is low, is traded at a high price because of its usefulness in hedging market risk. On the other hand, the additional probability weighting $\exp \left(-\tau F_{Z}(Z)\right)$ that is introduced here is due to the probability distortions and associates the price of a position with the rank of the outcome of $Z$, in the set of possible outcomes. For this factor, the absolute value of $Z$ is not of interest, but rather the ranking of its possible outcomes, induced by the application of its cumulative distribution function, $F_{Z}$. That the price density is a decreasing function of $F_{Z}(Z)$ has again the interpretation that a position, which is likely to assume a high value when $F_{Z}(Z)$ is low, is traded at a high price because of its usefulness in hedging. However, hedging now takes place not with respect to the absolute level of market risk, but with respect to its rank among all possible outcomes; in that sense this is not hedging against losses, but hedging against scenarios.

The effect of a change in collective risk or ambiguity aversion is not easily glanced from (24). However, by expanding $\pi(X)$ for small values of $\rho$ and $\tau$, and considering only first order terms one obtains $\pi(X) \approx E[X]-\rho \operatorname{Cov}(X, Z)-$ $\tau \operatorname{Cov}\left(X, F_{Z}(Z)\right)$, which could in effect be viewed as a generalised version of the Capital Asset Pricing Model formula. From that it is apparent that increases in the collective risk or ambiguity aversion coefficients lead to decreases in the price of an instrument that is positively correlated to the aggregate risk. This is equivalent to saying that asset returns will increase and that insurance will become more expensive. Intuitively this makes sense as one would expect increased risk and ambiguity aversion would drive agents to require higher returns on their risky investments. Moreover, the parameter $\tau$ introduces an increase in prices not captured by classical utility-based models; ambiguity aversion can thus be viewed as a possible explanation for the 'equity premium puzzle' (Mehra and Prescott, 1985). In the CEU interpretation of distortion functions, Knightian uncertainty is the reason behind such an increase in market prices, a point already argued by Epstein and Wang (1994). Our framework additionally shows that rank-dependence of preferences may also be the reason behind asset returns that exceed utility theory's predictions.

We can now explicitly calculate the agents' final positions $Y_{1}, Y_{2}, \ldots, Y_{n}$.

Proposition 2. In the risk exchange with exponential utilities and distortions the risk allocations $Y_{1}, Y_{2}, \ldots, Y_{n}$ are given by

$$
Y_{i}=\frac{\rho}{\rho_{i}}(Z-\pi(Z))+\frac{\tau-\tau_{i}}{\rho_{i}}\left(F_{Z}(Z)-\pi\left(F_{Z}(Z)\right)\right)+\pi\left(X_{i}\right), \quad i=1,2, \ldots, n .
$$

Proof: Equations (25), (24) yield:

$$
\begin{aligned}
e^{-\rho_{i} Y_{i}} \frac{e^{-\tau_{i} U}}{E\left[e^{-\tau_{i} U}\right]} & =\lambda_{i} \frac{e^{-\rho Z-\tau U}}{E\left[e^{-\rho Z-\tau U}\right]} \Rightarrow Y_{i} \\
& =\frac{\rho}{\rho_{i}} Z+\frac{\tau-\tau_{i}}{\rho_{i}} U-\frac{1}{\rho_{i}} \ln \left(\lambda_{i} \frac{e^{-\tau_{i} U}}{E\left[e^{-\rho Z-\tau U}\right]}\right)
\end{aligned}
$$


From the constraint in (11) we obtain (the equality being a consequence of the strict positivity of the lagrangian multiplier $\left.\lambda_{i}(14)\right)$ :

$$
\begin{gathered}
\pi\left(Y_{i}\right)=\pi\left(X_{i}\right) \Rightarrow \\
-\frac{1}{\rho_{i}} \ln \left(\lambda_{i} \frac{e^{-\tau_{i} U}}{E\left[e^{-\rho Z-\tau U}\right]}\right)=-E\left[\left(\frac{\rho}{\rho_{i}} Z+\frac{\tau-\tau_{i}}{\rho_{i}} U\right) \zeta\right]+E\left[X_{i} \zeta\right] .
\end{gathered}
$$

Substituting (33) in (32) yields $Y_{i}$.

It can easily be seen that, as expected, the share of the aggregate risk that the $i$ th agent holds after the exchange decreases as his risk and ambiguity aversion coefficients increase. Specifically, $Y_{i}$ depends on how they compare with the corresponding collective risk and ambiguity aversions. Note that the risk allocation $Y_{i}$ consists of two terms: the first is a proportional share of the aggregate risk $Z$, due to risk aversion, and the second a proportional share of $F_{Z}(Z)$, due to ambiguity aversion. Regarding the latter, it depends on the difference between the individual and collective ambiguity aversions $\tau-\tau_{i}$; if they are equal it vanishes. Furthermore, the aggregate traded share of $F_{Z}(Z)$ is zero, since $\sum_{j=1}^{n} \frac{\tau-\tau_{j}}{\rho_{j}}=0$. In the context of ambiguity, we can interpret the trading in $F_{Z}(Z)$ as agents' with diverging beliefs betting against each other. In that sense, ambiguity can be a source of trading, as discussed in Billot et al. (2000). It is noted that betting behaviour has been produced by older models, such as Wilson's (1968), which considers diverging beliefs in the context of Savage expected utility. The above results show that rank dependence of preferences as well as diverging beliefs can cause incentives for betting. The reason behind this is that under the rank-dependent model agents' probabilities are re-weighted by $h_{i}^{\prime}\left(S_{Y_{i}}\left(Y_{i}\right)\right)$. Mathematically this corresponds to a change of probability measure and is thus akin to diverging beliefs.

\subsection{Solution for the general case}

We now proceed with the calculation of the equilibrium price density, for the case where agents' preferences are characterised by more general utility and distortion functions.

In Section 3.2 it was shown that at equilibrium the agents' final positions $Y_{i}$ will be comonotonic to each other, as well as to their sum $Z$. Thus for each $i=1, \ldots, n, Y_{i}$ can be written as an increasing function $\psi_{i}$ of $Z, Y_{i}=\psi_{i}(Z)$. From equation (14) it is then apparent that the price density $\zeta$ will be a decreasing function $\phi$ of $Z, \zeta=\phi(Z)$. Thus, we can rewrite the condition for equilibrium (14) as:

$$
u_{1}^{\prime}\left(\psi_{i}(Z)\right) h_{1}^{\prime}\left(S_{Z}(Z)\right)=\lambda_{i} \phi(Z), \quad i=1, \ldots, n .
$$


Denoting the $i$ th agent's risk aversion and ambiguity aversion functions by $\rho_{i}(x)$ and $\tau_{i}(s)$ respectively,

$$
\rho_{i}(x)=\frac{u_{i}^{\prime \prime}(x)}{u_{i}^{\prime}(x)}, \tau_{i}(s)=\frac{h_{i}^{\prime \prime}(s)}{h_{i}^{\prime}(s)}
$$

we proceed with the following definition:

Definition 4. In the risk exchange with general utility and distortion functions, the collective risk aversion, $\rho(x)$, and the collective ambiguity aversion, $\tau(S)$, are defined by the equations:

$$
\rho(Z)=\left(\sum_{j=1}^{n} \frac{1}{\rho_{j}\left(\psi_{j}(Z)\right)}\right)^{-1}, \tau\left(S_{Z}(Z)\right)=\rho(Z) \sum_{j=1}^{n} \frac{\tau_{j}\left(S_{Z}(Z)\right)}{\rho_{j}\left(\psi_{j}(Z)\right)} .
$$

Again the formula for the collective risk aversion goes back to Bühlmann (1984) and Borch (1985), while the formula for $\tau(S)$ is being introduced in this paper.

As before, the calculation of the equilibrium price density, $\zeta$, relies on the constructs of collective risk and ambiguity aversion.

Proposition 3. In the risk exchange with general utilities and distortions the equilibrium price density, $\zeta$, has the form:

$$
\zeta=\frac{e^{-\int_{-\infty}^{Z} \rho(x) d x-\int_{0}^{F_{Z}(Z)} \tau(1-y) d y}}{E\left[e^{-\int_{-\infty}^{Z} \rho(x) d x-\int_{0}^{F_{Z}(Z)} \tau(1-y) d y}\right]} .
$$

Proof: Taking the logarithmic derivative of both sides of (34) (which will exist because of our smoothness assumptions) yields

$$
\begin{gathered}
\frac{\partial \ln \left(u_{i}^{\prime}\left(\psi_{i}(Z)\right) h^{\prime}\left(S_{Z}(Z)\right)\right)}{\partial Z}=\frac{\partial \ln \left(\lambda_{i} \phi(Z)\right)}{\partial Z} \Rightarrow \\
\frac{u_{i}^{\prime \prime}\left(\psi_{i}(Z)\right)}{u_{i}^{\prime}\left(\psi_{i}(Z)\right)} \psi_{i}^{\prime}(Z)-\frac{h_{i}^{\prime \prime}\left(S_{Z}(Z)\right)}{h_{i}^{\prime}\left(S_{Z}(Z)\right)} f_{Z}(Z)=\lambda_{i} \frac{\phi^{\prime}(Z)}{\phi(Z)},
\end{gathered}
$$

where $f_{Z}$ is the probability density function of $Z$. Substituting the $i$ th agent's risk and ambiguity aversions (35) in eq. (38) yields

$$
\psi_{i}^{\prime}(Z)=-\frac{1}{\rho_{i}\left(\psi_{i}(Z)\right)} \lambda_{i} \frac{\phi^{\prime}(Z)}{\phi(Z)}-\frac{\tau_{i}\left(S_{Z}(Z)\right)}{\rho_{i}\left(\psi_{i}(Z)\right)} f_{Z}(Z) .
$$


Differentiating the clearing condition (12) yields $\sum_{j=1}^{n} \psi_{j}^{\prime}(Z)=1$. Thus, summing over $i$ in (39) using the definition of the collective risk and ambiguity aversions, $\rho(x)$ and $\tau(S)$ we obtain the ordinary differential equation

$$
\begin{aligned}
\frac{\phi^{\prime}(Z)}{\phi(Z)} & =\lambda_{i}^{-1}\left(-\rho(Z)-\tau\left(S_{Z}(Z)\right) f_{Z}(Z)\right) \Rightarrow \phi(Z) \\
& =K e^{-\int_{-\infty}^{Z} \rho(x) d x-\int_{-\infty}^{Z} \tau\left(S_{Z}(x)\right) f_{Z}(x) d x},
\end{aligned}
$$

for some constant $K$. Eq. (40) and the condition $E[\phi(Z)]=E[\zeta]=1$ yield formula (37) for the price density.

This price density is a generalisation of the formula obtained by Bühlmann (1984). Again the market's ambiguity aversion introduces an additional weighting factor, $\exp \left\{-\int_{0}^{F_{Z}(Z)} \tau(1-y) d y\right\}$. The discussion in the previous sections of the influence of ambiguity aversion on the pricing of risk retains its validity in this, more general setting. In the case of exponential utility and distortion functions studied in the previous section, the market as well as the individual risk and ambiguity aversion functions are constant and it is easily seen that equation (37) reduces to (24).

We now obtain an expression for the risk allocations $\psi_{i}(Z)=Y_{i}$.

Proposition 4. In the risk exchange with general utilities and distortions the risk allocations $Y_{1}, Y_{2}, \ldots, Y_{n}$ are given by

$$
Y_{i}=\left(u_{i}^{\prime}\right)^{-1} \circ \exp \left\{-\int_{-\infty}^{Z} \rho(x) d x-\int_{0}^{F_{Z}(Z)}\left(\tau(1-y)-\tau_{i}(1-y)\right) d y-K\right\},
$$

where the constant $K$ is determined by the budget condition $\pi\left(Y_{i}\right)=\pi\left(X_{i}\right)$.

Proof: As mentioned in Section 2.2, from the quantities $\rho(Z)$ and $\tau\left(S_{Z}(Z)\right)$ we can determine unique corresponding utility and distortion functions $u$ and $h$ respectively (up to a normalisation of $u$ ). We can rewrite the price density (37) as

$$
\phi(Z)=\frac{u^{\prime}(Z) h^{\prime}\left(S_{Z}(Z)\right)}{E\left[u^{\prime}(Z) h^{\prime}\left(S_{Z}(Z)\right)\right]}
$$

which yields

$$
\begin{aligned}
\frac{\phi^{\prime}(Z)}{\phi(Z)} & =\frac{u^{\prime \prime}(Z) h^{\prime}\left(S_{Z}(Z)\right)-u^{\prime}(Z) h^{\prime \prime}\left(S_{Z}(Z)\right) f_{Z}(Z)}{u^{\prime}(Z) h^{\prime}\left(S_{Z}(Z)\right)} \\
& =-\rho(Z)-\tau(Z) f_{Z}(Z)
\end{aligned}
$$


Substituting the term $\frac{\phi^{\prime}(Z)}{\phi(Z)}$ in equation (39) results in the differential equation

$$
\psi_{i}^{\prime}(Z) \rho_{i}\left(\psi_{i}(Z)\right)=\rho(Z)+\tau\left(S_{Z}(Z)\right)-\tau_{i}\left(S_{Z}(Z)\right) f_{Z}(Z)
$$

The definition of the risk aversion coefficient yields $\psi_{i}^{\prime}(Z) \rho_{i}\left(\psi_{i}(Z)\right)=-\partial \ln$ $u_{i}^{\prime}\left(\psi_{i}(Z)\right) / \partial Z$. Hence (44) yields

$$
u_{i}^{\prime}\left(\psi_{i}(Z)\right)=\exp \left\{-\int_{-\infty}^{Z} \rho(x) d x-\int_{-\infty}^{Z}\left(\tau\left(S_{Z}(s)\right)-\tau_{i}\left(S_{Z}(s)\right)\right) d s-K\right\},
$$

whence (41) follows.

From (41) and (44) we see that, as in the previous section, $Y_{i}$ depends on how the $i$ th agent's risk and ambiguity aversions compare to the collective ones. Observe that in this more general case the $Y_{i}$ 's do not consist of proportional shares of $Z$ and $F_{Z}(Z)$, but are non-linear functions thereof. The trading in $F_{Z}(Z)$ can again be interpreted as betting behaviour, similarly to the previous section. We note that (41) does not provide a closed form solution for $Y_{i}$, as the collective risk aversion $\rho$ does in general depend on the $Y_{i}$ 's.

\subsection{Ambiguity, (in)determinacy and betting}

In the previous sections, agents' distortion functions have been taken to represent either rank-dependent preferences or ambiguity with respect to probability, without any distinction being made between these two very different cases. As mentioned earlier, an important difference is the role played by the probability measure $\mathbb{P}_{0}$. In the rank dependent case $\mathbb{P}_{0}$ is a 'real-world' probability measure, known by all agents, and the distortion functions affect the subjective perception of this probability by the agents. Thus the equilibrium prices and allocations calculated in previous sections completely determine the equilibrium of the risk exchange.

On the other hand, if distortion functions are interpreted as inducing sets of probability measures representing Knightian uncertainty, then $\mathbb{P}_{0}$ is just a reference measure, arbitrarily drawn from a set of probability measures with the same null-sets. Agents' probabilistic beliefs are represented by supermodular capacities, which can be obtained by distorting a probability measure from that set, with the distortion functions generally depending on the reference measure chosen. This means that the analysis carried out in the previous sections could be repeated for a different reference measure, say $\overline{\mathbb{P}}_{0}$. Given that there is no conceivable mechanism by which agents would agree on a reference measure before trading, if the equilibrium calculated under $\overline{\mathbb{P}}_{0}$ is different than the one under $\mathbb{P}_{0}$, then the conclusion must be that, in the case of ambiguity, equilibria are indeterminate. 
To show that this is actually the case, we can restrict ourselves to the case where utilities are exponential. Consider an agent for whom $h_{i}\left(\mathbb{P}_{0}\right)=\bar{h}_{i}\left(\overline{\mathbb{P}}_{0}\right)$. As $h_{i}, \bar{h}_{i}$ will in general be different, the same will hold for the associated ambiguity aversions, i.e. $\tau_{i}(s) \neq \bar{\tau}_{i}(s)$. Note that $F_{Z}(Z)$ will be uniformly distributed under both measures and that the individual and collective risk aversions, $\rho_{j}$, $j=1, \ldots, n$ and $\rho$, will be independent of the equilibrium risk allocation and thus not depend on the reference measure. From (41), it can now be seen that, if the $\tau_{i}$ 's change, other things being equal, the equilibrium allocations change. Thus ambiguity is a source of indeterminacy.

Consider the case of all agents' beliefs being represented by the same capacity. It is then obvious that for any reference measure it is $\tau_{i}(s)=\tau(s) \forall s \in[0,1]$, $\forall i=1, \ldots, n$. From (41) it can be seen that that this makes the risk allocations independent of the reference probability measure $\mathbb{P}_{0}$. Thus, in the case of a shared capacity the indeterminacy of the risk allocations vanishes. Furthermore, the part of the allocation which is due to ambiguity aversion, is increasing in the difference between individual and collective risk aversion, that is, in the degree to which an agents' beliefs diverge from the average. As discussed in Sections 3.3 and 3.4, diverging beliefs are a cause of trading, in the sense that it motivates agents to bet against each other. If all agents' ambiguity is represented by the same capacity, then the part of the allocation depending on ambiguity aversion vanishes for all agents. This is consistent with Chateauneuf et al. (2000), who showed that risk allocations in the case of a common capacity are the the same as von Neumann-Morgenstern ones. Thus, under homogeneity of beliefs, betting ceases. On the other hand, equilibrium prices always depend on collective ambiguity aversion, as can be seen from (37). Therefore even in the case where agents have the same beliefs, equilibrium prices are indeterminate.

Agents' betting behavior under diverse levels of ambiguity, allows for a further interpretation of the comonotonicity of allocations. When allocations are comonotonic, agents use the same probability distribution at equilibrium. ${ }^{4}$ Thus, even though equilibrium does not lead to the resolution of ambiguity, it yields an implicit agreement between agents, since they behave as if they were using the same unique probability distribution.

Finally, we note that if the aggregate risk is zero, i.e. $Z$ is a constant, the risk allocations are also constant, meaning that the lack of aggregate risk yields full insurance allocations, regardless of ambiguity. This is consistent with Billot et al. (2000).

\subsection{On collective preferences}

In the equilibrium models discussed in previous sections, agents' risk allocations, $Y_{i}, i=1, \ldots, n$, and the price density, $\zeta$, have been found to be functions only of the aggregate market risk, $Z$. Furthermore, the price functional depends only

4 The representation of Choquet integrals of comonotonic random variables through the same probability measure follows from Proposition 10.1 in Denneberg (1994). 
on $Z$ and the collective risk and ambiguity aversions, $\rho(x)$ and $\tau(S)$ respectively. The technique of defining collective preferences is not a novelty; it is closely related to the device of the 'representative agent' often employed in the economics literature, while the aggregation of preferences has been proposed as a way of solving equilibrium models by Borch (1962), Wilson (1968), Rubinstein (1974) and Bühlmann (1980, 1984).

A new element introduced in this paper has been the definition of aggregate preferences in the cases of RDEU and CEU, using what we called 'collective ambiguity aversion'. From the definitions of collective ambiguity aversion (23), (36) it can be seen that it does not only depend on the ambiguity aversions of the individual agents, but also on their risk aversions. In fact the collective ambiguity aversion $\tau$ is determined as the average of individual ambiguity aversions $\tau_{i}$, weighted by the risk tolerances $1 / \rho_{i}$. This could appear problematic. It is however justifiable, considering that collective preferences depend on the allocation of risks, which in turn depend on the utilities as well as the distortion functions of agents. From equations (31) and (44) it can be seen that the allocations of risk (i.e. functions of both $Z$ and $F_{Z}(Z)$ ) to the agents are dependent on the risk tolerances $1 / \rho_{i}$ (in the exponential case the risk allocations are actually proportional to the risk tolerances). It is reasonable that an agent who ends up buying a larger share of the market risk will also have a larger effect on aggregate preferences, as the definition of collective ambiguity aversion implies.

It is apparent from the definitions (23) and (36) that the collective risk aversion is lower than that of any agent in the exchange. This can be interpreted as reflecting the reduction in risk that the possibility of risk sharing and diversification through the exchange entails. A way to see this is to observe that the reduction of agents' risk aversion caused by their participation in the market also results in a reduction of the price of insurance for a risk. On the other hand, such reduction does not take place in the case of ambiguity aversion. As collective ambiguity aversion is a weighted average of agents' individual ambiguity aversions, it might be greater or smaller than the one of an agent. This effect can be better understood through the interpretation of the convex probability distortion functions as reflecting ambiguity with respect to probability, in the context of Knightian uncertainty. If all agents are uncertain about the probabilities of future events, there is no reason why adding traders to the market (provided they are not better informed than the rest) should reduce such uncertainty; ambiguity cannot thus be 'diversified away'.

From the collective risk and ambiguity aversions we can determine respectively a utility $u$ and a distortion $h$, as well as the corresponding preference functional $V$. Consider now the representative agent, holding $Z$ and with preferences characterised by $V$. We define the indifference price of a position $X$ to the collective, $\pi_{\text {ind }}(X ; Z)$, as the solution of $\left.V\left(Z-X+\pi_{\text {ind }}(X ; Z)\right)\right)=V(Z)$ and the marginal cost of $X$ to the collective as:

$$
M C(X ; Z)=\left.\frac{\partial \pi_{\text {ind }}(\beta X ; Z)}{\partial \beta}\right|_{\beta=0} .
$$


It can then be shown, using the same techniques as in the proof of Lemma 6, that the marginal cost of $X$ to the collective equals the equilibrium price of $X$ :

$$
\begin{aligned}
M C(X ; Z) & =E\left[X \frac{u^{\prime}(Z) h^{\prime}\left(S_{Z}(Z)\right)}{E\left[u^{\prime}(Z) h^{\prime}\left(S_{Z}(Z)\right)\right]}\right] \\
& =E\left[X \frac{e^{-\int_{-\infty}^{Z} \rho(x) d x-\int_{0}^{F_{Z}(Z)} \tau(1-y) d y}}{E\left[e^{-\int_{-\infty}^{Z} \rho(x) d x-\int_{0}^{F_{Z}(Z)} \tau(1-y) d y}\right]}\right] .
\end{aligned}
$$

This is, of course, just another way to say that at equilibrium risks are priced under collective preferences.

Finally, we note that an alternative interpretation of collective preferences is to view the risk exchange at equilibrium as a pooling arrangement, where agents pool their initial endowments, $X_{i}, i=1, \ldots, n$, and thereafter share the aggregate risk $Z$ by buying their final positions $Y_{i}$ from the pool, according to an agreed price mechanism. The analogy between risk exchange and risk pooling has already been observed by Borch (1962), who commented on the possibility of applying cooperative game theory to the problem. The marginal cost price mechanism, which yields equilibrium prices, has a theoretical justification in the context of cooperative games, as it belongs to the class of semi-values (Dubey et al., 1981), while it can also be derived from a set of economically motivated axioms (Samet and Tauman, 1982). The relationship between pooling (cooperative risk sharing) and trading (competitive risk sharing) follows from the comonotonicity of the risk allocations at equilibrium. Comonotonicity will make all agents' fortunes move in the same direction, as it would have been, were they pooling their risks. It is thus the efficient spreading of risk that comonotonicity implies, which makes cooperative and competitive economic behavior in some sense equivalent.

\section{ConcLusions}

Equilibria in risk exchanges were studied, when agents' decision making takes place under distorted probabilities. Distorted probabilities are used to represent the preference functionals of Rank-Dependent and Choquet Expected Utilities, which have emerged in recent years as important correctives to the Expected Utility paradigm. Explicit formulae for the state-price density and risk allocations were obtained, thus generalizing results obtained by Borch (1962) and Bühlmann $(1980,1984)$, who considered expected utility preferences.

The solution of the equilibrium models utilises the construction of collective preferences. A 'collective ambiguity aversion' coefficient was introduced in the paper to characterise the effect of probability distortion on aggregated preferences. It was shown that due to probability distortion an additional term 
appears in both the state price density and the allocations of risk to insurers. The change in risk allocations shows that probability distortion gives to agents incentives to trade, not only in order to share the aggregate risk, but also to bet against each other. In the context of CEU, such behaviour can be attributed to diverging beliefs about the probabilities of future states of the world.

While the RDEU and CEU preference models present some formal similarities, they are quite different in terms of the phenomena they seek to explain. Thus, while in the case of rank-dependent preferences a known probability measure is distorted by agents' perception, in the case of ambiguity a distorted reference measure is used to represent ambiguous beliefs. Choice of different reference measures yields different equilibria; hence one concludes that equilibria under ambiguity are indeterminate.

\section{A. TWO LEMMAS}

Let an agent's preferences be characterised by a utility function $u$ and a distortion function $h$. Here we obtain two results concerning the operator $V_{u, h}$ that are used extensively in the paper.

Lemma 5. For every $X \in X, V_{u, h}(X)=E\left[u(X) h^{\prime}\left(S_{X}(X)\right)\right]$.

Proof: The Choquet integral (1) of $u(X)$ with respect to the supermodular set function $h\left(\mathbb{P}_{0}\right)$, admits the following quantile representation (Denneberg, 1994):

$$
V_{u, h}(X)=\int_{0}^{1} G_{u(X)}^{-1}(t) d t,
$$

where $G_{u(X)}^{-1}(t)$ is the (generalised) inverse of the (decumulative) distribution function of $u(X)$ under $h\left(\mathbb{P}_{0}\right), G_{u(X)}(x)=h\left(\mathbb{P}_{0}(u(X)>x)\right)=h\left(S_{u(X)}(x)\right)$. Since the functions $S_{X}, h, u, S_{u(X)}$ are strictly monotonic, $G_{u(X)}^{-1}(t)=S_{u(X)}^{-1}\left(h^{-1}(t)\right)=$ $u\left(S_{X}^{-1}\left(h^{-1}(t)\right)\right) . V_{u, h}(X)$ can then be written as:

$$
V_{u, h}(X)=\int_{0}^{1} u\left(S_{X}^{-1}\left(h^{-1}(t)\right)\right) d t
$$

By performing the change of variable $t=h\left(S_{X}(x)\right)$, we obtain:

$$
V_{u, h}(X)=\int_{+\infty}^{-\infty} u(x) d h\left(S_{X}(x)\right)=\int_{+\infty}^{-\infty} u(x) h^{\prime}\left(S_{X}(x)\right)\left(-f_{X}(x)\right) d x .
$$

Thus $V_{u, h}(X)=E\left[u(X) h^{\prime}\left(S_{X}(X)\right)\right]$. 
Corollary 1. $R_{h}(X)=-E\left[X h^{\prime}\left(S_{X}(X)\right)\right]$.

Lemma 6. Let $X, N \in X$ and $\beta \in \mathbb{R}$. Then $V_{u, h}(X+\beta N)$ is differentiable with respect to $\beta$ and the partial derivative equals:

$$
\frac{\partial}{\partial \beta} V_{u, h}(X+\beta N)=E\left[N u^{\prime}(X+\beta N) h^{\prime}\left(S_{X+\beta N}(X+\beta N)\right)\right] .
$$

Proof: As in the proof of the previous lemma, we use the quantile representation of the Choquet integral:

$$
V_{u, h}(X+\beta N)=\int_{0}^{1} u\left(S_{X+\beta N}^{-1}\left(h^{-1}(t)\right)\right) d t=\int_{0}^{1} u\left(S_{X+\beta N}^{-1}(s)\right) d h(s) .
$$

Assuming continuity of conditional densities, Tasche (2000) shows that:

$$
\frac{\partial}{\partial \beta} S_{X+\beta N}^{-1}(s)=E\left[N \mid X+\beta N=S_{X+\beta N}^{-1}(s)\right] .
$$

Thus, the derivative of $V_{u, h}(X+\beta N)$ with respect to $\beta$ is:

$$
\begin{gathered}
\frac{\partial}{\partial \beta} V_{u, h}(X+\beta N)=\int_{0}^{1} u^{\prime}\left(S_{X+\beta N}^{-1}(s)\right) \frac{\partial}{\partial \beta} S_{X+\beta N}^{-1}(s) d h(s)= \\
\int_{0}^{1} u^{\prime}\left(S_{X+\beta N}^{-1}(s)\right) E\left[N \mid X+\beta N=S_{X+\beta N}^{-1}(s)\right] d h(s) \stackrel{\left(S_{X+\beta N}^{-1}(s)=y\right)}{=} \\
\int_{+\infty}^{-\infty} u^{\prime}(y) E[N \mid X+\beta N=y] d h\left(S_{X+\beta N}(y)\right)= \\
\int_{+\infty}^{-\infty} u^{\prime}(y)\left(\int_{-\infty}^{+\infty} n \frac{f_{N, X+\beta N}(n, y)}{f_{X+\beta N}(y)} d n\right) h^{\prime}\left(S_{X+\beta N}(y)\right)\left(-f_{X+\beta N}(y)\right) d y= \\
\int_{-\infty}^{+\infty} \int_{-\infty}^{+\infty} n u^{\prime}(y) h^{\prime}\left(S_{X+\beta N}(y)\right) f_{N, X+\beta N}(n, y) d n d y .
\end{gathered}
$$

So, we finally obtain:

$$
\frac{\partial}{\partial \beta} V_{u, h}(X+\beta N)=E\left[N u^{\prime}(X+\beta N) h^{\prime}\left(S_{X+\beta N}(X+\beta N)\right)\right] .
$$

\section{Corollary 2.}

$$
\frac{\partial}{\partial \beta} R_{h}(X+\beta N)=-E\left[N h^{\prime}\left(S_{X+\beta N}(X+\beta N)\right)\right] .
$$




\section{REFERENCES}

AASE, K. (1993) Equilibrium in a reinsurance syndicate; existence, uniqueness and characterisation. ASTIN Bulletin 23(2), 185-211.

Aase, K. (2002) Perspectives of Risk Sharing. Scandinavian Actuarial Journal 2, 73-128.

Artzner, P., Delbaen, F., Eber, J.-M., and Heath, D. (1999) Coherent measures of risk. Mathematical Finance 9(3), 203-228.

Aubin, J-P. (1981) Cooperative Fuzzy Games. Mathematics of Operations Research 6(1), 1-13.

Aumann, R.J. and ShaPleY, L.S. (1974) Values of Non-Atomic Games. Princeton University Press, Princeton.

Basle Committee on Banking Supervision (2003) The New Basel Capital Accord.

Billot, A., Chateauneuf, A., Gilboa, I. and Tallon, J.-M. (2000) Sharing Beliefs: Between Agreeing and Disagreeing. Econometrica 68(3), 685-694.

Borch, K. (1962) Equilibrium in a Reinsurance Market. Econometrica 30(3), 424-444.

Borch, K. (1985) A Theory of Insurance Premiums. The Geneva Papers of Risk and Insurance, 192-208.

Bühlmann, H. (1980) An Economic Premium Principle. ASTIN Bulletin 11(1), 52-60.

Bühlmann, H. (1984) The General Economic Premium Principle. ASTIN Bulletin 14(1), 13-21.

CARlier, G. and DANA, R.A. (2002) Core of convex distortions of a probability. Journal of Economic Theory 113(2), 199-222.

Chateauneuf, A., Dana, R.A. and Tallon, J.-M. (2000) Optimal Risk-Sharing Rules and Equilibria with Choquet-Expected-Utility. Journal of Mathematical Economics 34, 191-214.

Chen, Z. and Epstein, L. (2002) Ambiguity, risk, and asset returns in continuous time. Econometrica 70(4), 1403-1443.

Chew, S.H., Karni, E. and SAfra, Z. (1987) Risk Aversion in the Theory of Expected Utility with Rank Dependent Probabilities. Journal of Economic Theory 42, 370-381.

Choquet, G. (1953) Theory of capacities. Annales de l'Institut Fourier 5, 131-295.

DAnA, R-A. (2002) On Equilibria when Agents have Multiple Priors. Annals of Operations Research 114, 105-115.

Denneberg, D. (1990) Distorted Probabilities and Insurance Premiums. Methods of Operations Research 63, 3-5.

Denneberg, D. (1994) Non-additive Measure and Integral. Kluwer Academic Publishers, Dordrecht.

Dhaene, J., Denuit, M., Goovaerts, M.J., KaAs, R. and Vyncke, D. (2002) The Concept of Comonotonicity in Actuarial Science and Finance: Theory. Insurance: Mathematics and Economics 31(1), 3-33.

Dubey, P., Neyman, A. and Weber, R.J. (1981) Value Theory Without Efficiency. Mathematics of Operations Research 6, 122-128.

Duffie, D. (1996) Dynamic Asset Pricing Theory. Princeton University Press, Princeton.

EpsteIN, L.G. and Wang, T. (1994) Intertemporal asset pricing under Knightian uncertainty. Econometrica 62(3), 283-322.

GilboA, I. (1985) Subjective Distortions of Probabilities and Non-additive Probabilities. Preprint, The Foerder Institute of Economic Research, Tel Aviv University.

GilboA, I. and Schmeidler, D. (1989) Maxmin Expected Utility with a Non-unique Prior. Journal of Mathematical Economics 18, 141-153.

Knight, F.H. (1921) Risk, Uncertainty and Profit. Houghton Mifflin, Boston.

Landsberger, M. and Meilijson, I. (1994) Co-monotone Allocations, Bickel-Lehmann dispersion and the Arrow-Pratt measure of risk aversion. Annals of Operations Research 52, $97-$ 106.

Mehra, R. and Prescott, E. (1985) The Equity Premium: A Puzzle. Journal of Monetary Economics 15, 145-161.

Quiggin, J. (1982) A Theory of Anticipated Utility. Journal of Economic Behavior and Organization 3, 323-343.

Quiggin, J. (1993) Generalized Expected Utility Theory: The Rank-dependent Model. Kluwer Academic Publishers, Boston.

Rothschild, M. and Stiglitz, J. (1970) Increasing Risk: I. A Definition. Journal of Economic Theory 2, 225-243 
Rubinstein, M. (1974) An Aggregation Theorem for Securities Markets. Journal of Financial Economics 1, 225-244.

Samet, D., and Tauman, Y. (1982) The Determination of Marginal Cost Prices under a Set of Axioms. Econometrica 50(4), 895-909.

SchmeIdLeR, D. (1989) Subjective Probability and Expected Utility without Additivity. Econometrica 57(3), 571-587.

Tasche, D. (2000) Conditional Expectation as Quantile Derivative. Preprint, TU Munich.

Tsanakas, A. and Desli, E. (2003) Risk measures and theories of choice. British Actuarial Journal 9(4), 959-991.

von Neumann, J. and Morgenstern, O. (1944) Theory of Games and Economic Behavior. Princeton University Press, Princeton.

WANG, S.S. (1996) Premium Calculation by Transforming the Premium Layer Density. ASTIN Bulletin, 26(1), 71-92.

WiLSON, R. (1968) The Theory of Syndicates. Econometrica 36(1), 119-132.

YAARI, M. (1987) The Dual Theory of Choice under Risk. Econometrica 55(1), 95-115.

ANDREAS TSANAKAS ${ }^{*}$

Market Risk and Reserving Unit

Lloyd's of London

One Lime Street

London EC3M 7 HA

United Kingdom

E-mail: andreas.tsanakas@lloyds.com

Nicos Christofides

Centre for Quantitative Finance

Imperial College London

Exhibition Road

London SW7 $2 A Z$

United Kingdom

E-mail:n.christofides@imperial.ac.uk

* The views expressed in this paper are solely those of the authors and do not necessarily reflect the views of Lloyd's of London. 